\title{
Eye Opener to EtOH Ablation for Juxta-Cardiac Hepatocellular Carcinoma
}

\author{
Erik Soule Sanjay Lamsal Chandana Lall Jerry Matteo \\ Department of Interventional Radiology, University of Florida, UF Health Jacksonville, \\ Jacksonville, FL, USA
}

\section{Keywords}

Hepatocellular carcinoma - EtOH ablation · Cirrhosis - Juxta-cardiac tumor

\begin{abstract}
Background: Hepatocellular carcinoma (HCC) is notoriously refractory to systemic chemotherapy, mandating an interventional approach. Mortality may be avoided by neutralizing rapidly growing tumors that approach the heart and major vessels. When the risk/benefit ratio of surgery is unacceptable, percutaneous ablation can achieve remarkable results. High volumes of flowing blood adjacent to the treatment area may impact the ability to reliably achieve an adequate ablation margin for modalities that rely on extreme temperatures to destroy malignant cells. Ethanol ablation is safe, efficacious, and unaffected by this "thermal sink" effect. This report describes a juxta-cardiac (JC) HCC in segment 4a measuring $35 \times 26$ $\mathrm{mm}$, which exhibited rapid growth until it was abutting the pericardium and $7.5 \mathrm{~mm}$ from the chamber of the right ventricle (RV). Methods: One 21-gauge needle was inserted using direct CT fluoroscopy into the center of the hepatic mass. In order to confirm the position of the needle, $0.5 \mathrm{~mL}$ of diluted Visipaque was injected. Then, under CT fluoroscopy guidance, a mixture of $1 \mathrm{~mL}$ of Ethiodol and $10 \mathrm{~mL}$ of $98 \%$ dehydrated alcohol was slowly injected into the mass. Results: Repeat CT scan 1 month post-ablation demonstrated decreased arterial enhancement and dense Ethiodol throughout the tumor consistent with ablation. Tumor size decreased to $30 \times 23 \mathrm{~mm}$ with a distance of $12.4 \mathrm{~mm}$ from the chamber of the RV. Conclusion: Pericardial involvement or large vessels near the treatment area may limit the use of thermal ablation techniques for JC HCC. Percutaneous, intratumoral ethanol injection provides safe and effective alternative that is not subject to the "thermal sink" effect.
\end{abstract}

(C) 2019 S. Karger AG, Basel 
Soule et al.: Eye Opener to EtOH Ablation for JC HCC

\section{Introduction}

Primary colon, breast, and lung malignancies frequently metastasize to the liver. Hepatocellular carcinoma (HCC), however, is the most common primary hepatic malignancy. HCC is the fifth most common cancer worldwide, and the fourth most common cause of cancerrelated deaths [1]. Unfortunately, because HCC is typically diagnosed late in the course of disease, the median survival after diagnosis ranges from 6 to 20 months [2]. Chronic hepatocellular injury, most commonly from hepatitis B virus, hepatitis $C$ virus, or all-cause cirrhosis drives development of HCC. Regeneration is accomplished by the release of bioactive factors that induce angiogenesis, hepatocyte remodeling, and cell survival. The continuously dividing cells experience genotoxic stress leading to accumulation of genetic mutations and dysplasia [3]. A universal feature of HCC is accumulation of chromosomal abnormalities leading to dysplasia, genomic instability, and malignant transformation.

HCC carcinogenesis occurs under chronic inflammatory conditions, such as those found in the livers of patients with alcoholic liver cirrhosis. Inflammation leads to focal hypoxia and necrosis, resulting in upregulation of vascular endothelial growth factor (VEGF) and other proangiogenic factors. These proangiogenic growth factors cause neovascularization, which is initiated by the destabilization of existing vessels causing "leakiness," further hypoxia, and increasing overexpression of VEGF in a positive feedback loop. Endothelial cell activation, recruitment, proliferation and organization into tumor vasculature are potentiated by this phenomenon. These abnormal vessels are stabilized by pericytes, allowing the tumor to receive blood supply [4]. The VEGF inhibitor sorafenib is the sole systemic agent with statistically significant efficacy against advanced HCC, touting a modest $44 \%$ improvement in overall survival [5]. This tyrosine kinase inhibitor is restricted to a minority of patients, as treatment may be precluded due to impaired liver function or advanced age [6]. Significant adverse effects include malaise, diarrhea, weight-loss, hand-foot syndrome, hypophosphatemia, neutropenia, and mucositis. The efficacy of sorafenib monotherapy has declined due to the emergence of tumor resistance [7].

Indeed, HCC is notoriously unresponsive to established systemic chemotherapies. Mechanistic understanding of the molecular determinants of HCC invasiveness may guide the development of future treatments. Epithelial-mesenchymal transition (EMT) is the main process by which HCC progresses, primarily through local invasion as opposed to distant metastasis. NF- $\mathrm{KB}$ is a transcription factor activated under inflammatory conditions, modulating DNA transcription to resist apoptosis as well as promoting EMT [8]. EMT is multifactorial: transforming growth factor-beta levels may be increased in patients with HCC, correlating with a worse prognosis. High levels of transforming growth factor-beta reduce the expression of E-cadherin at the cell surface, mRNA, and protein levels. E-cadherin is a transmembrane protein that acts as a cell-cell anchor; thus downregulation enables EMT and aggressive metastasis of HCC [9]. In the absence of effective systemic therapy against advanced HCC, surgical and ablative modalities are the mainstay of HCC management although the extent of cirrhosis may impact prognosis and treatment considerations [10]. After resection/ ablation, underlying chronic inflammatory conditions persist, often resulting in recurrent, multifocal disease in difficult to treat locations.

This report describes a 57-year-old male with past medical history of alcoholic liver cirrhosis who went on to develop multifocal HCC. He previously underwent 2 hepatic cryoablation procedures for HCC lesions in segment II as well as segment VIII. Subsequently he developed distant recurrences and underwent 2 transarterial chemoembolization procedures. On surveillance imaging the patient was noted to have developed a non-enhancing segment IVa lesion measuring $25 \times 20 \mathrm{~mm}$ along the cardiac border that demonstrated rapid growth (Fig. 1a-c). Over the course of 6 months it enlarged until it was abutting the peri- 

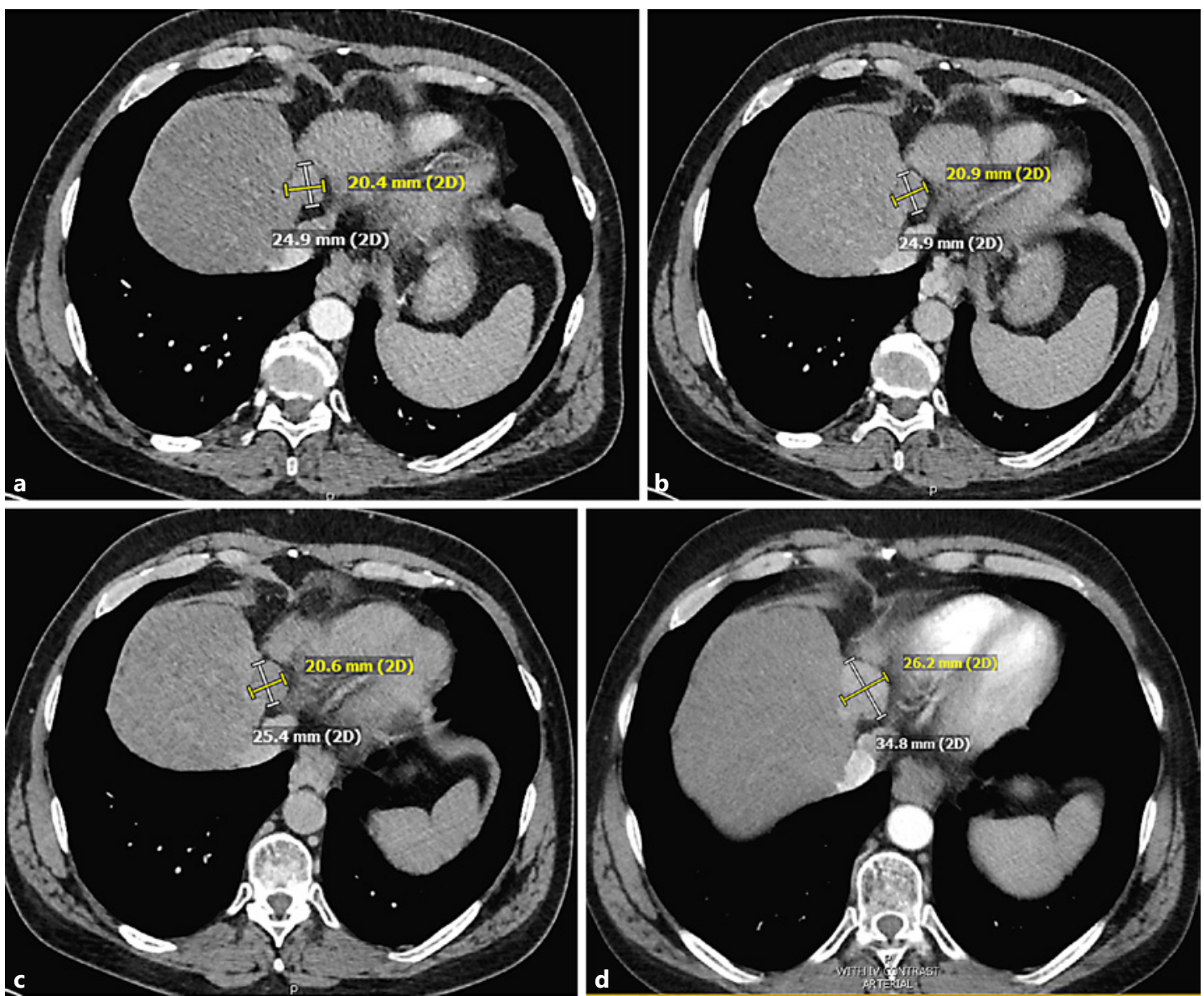

Fig. 1. Contrast-enhanced axial CT scan 9 months pre-procedure in arterial (a), portal venous (b), and delayed (c) phases demonstrating cirrhotic liver morphology and an HCC measuring $25 \times 20 \mathrm{~mm}$ in a juxtacardiac location within segment 4a. Contrast-enhanced axial CT scan 3 months pre-procedure in arterial phase demonstrating enhancement and rapid growth (d) of HCC to $35 \times 26 \mathrm{~mm}$.

cardium and measured $35 \times 26 \mathrm{~mm}$. It did show contrast enhancement in the arterial phase at that time (Fig. 1d). Continued growth in this area would imminently lead to right ventricle (RV) compression and compromised cardiopulmonary function.

\section{Materials and Methods}

Witnessed, informed written consent was obtained. Moderate sedation was utilized during the performance of this procedure with pre and post administration of sedation/analgesic patient evaluation, including the monitoring of the patient's cardiorespiratory function. An independent, trained observer assisted the physician in monitoring the patient. The patient was made to lie in a supine position on the CT gantry and prepped and draped in the usual sterile fashion. Maximal sterile barrier technique was used including cap, mask, sterile gown, sterile gloves, and a large sterile sheet plus hand hygiene and $2 \%$ chlorhexidine for cutaneous antisepsis.

Limited CT images were performed to delineate the left hepatic lobe segment IVa mass and for procedure planning. The skin was marked. Using CT guidance, one 21-gauge needle was inserted using direct CT fluoroscopy into the center of the hepatic mass. $0.5 \mathrm{~mL}$ of diluted Visipaque was injected to confirm the positioning. Then, under CT fluoroscopy guidance, a mixture of $1 \mathrm{~mL}$ of Ethiodol and $10 \mathrm{~mL}$ of $98 \%$ dehydrated 

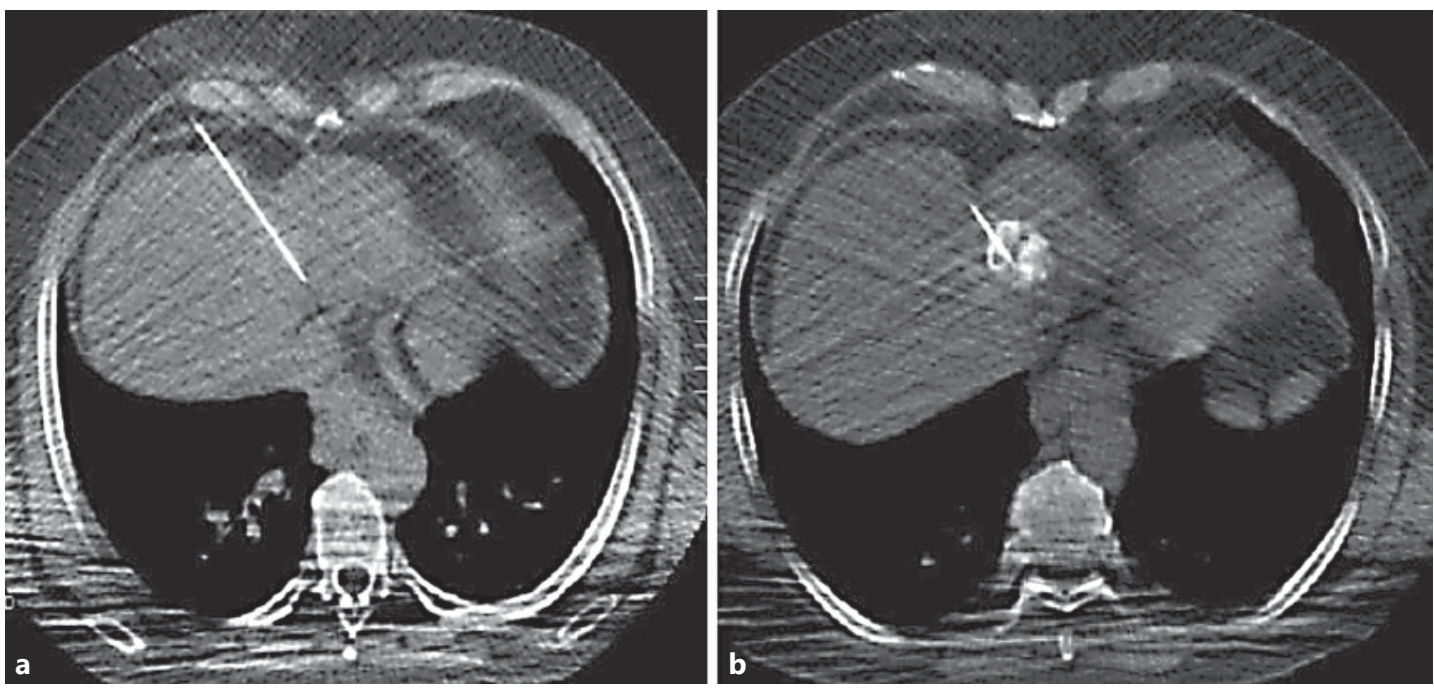

Fig. 2. a, b Intraoperative axial CT scan revealing a single needle inserted into the tumor, then injection of $10 \mathrm{~mL}$ 98\% EtOH with $1 \mathrm{~mL}$ of Ethiodol.

alcohol was slowly injected into the mass (Fig. 2a, b). A gold fiducial marker was placed into the lesion for potential future treatment planning. Then, the needle was removed.

Following the removal of the needle, limited CT was performed revealing hyperdense material within the region of the mass related to alcohol ablation. No complications or injury to surrounding organs were noted. Sterile dressing was then applied. The patient tolerated the procedure well without immediate complications. Following the completion of the procedure, the patient was transported to the interventional radiology recovery and monitoring area.

\section{Results}

Follow-up imaging revealed tumor ablation with shrinkage and retraction from the cardiac border (Fig. 3a-c). Prior to the procedure, the tumor measured $35 \times 26 \mathrm{~mm}$ and demonstrated arterial phase contrast enhancement. At that time, it was located a distance of $7.5 \mathrm{~mm}$ from the chamber of the RV and abutted the pericardium. One month post procedure, follow-up CT scan demonstrated Ethiodol throughout the mass and decreased arterial phase contrast enhancement. The size of the ablated tumor decreased to $30 \times 23 \mathrm{~mm}$. The distance of the ablated lesion to the chamber of the RV increased from 7.5 to $12.4 \mathrm{~mm}$ (Fig. 4a, b).

\section{Discussion}

Increased mortality due to invasion of vital structures such as major vessels and the heart itself may result from HCC growth in the deep hepatic segments. Although surgical resection is the first-line treatment for HCC, vascular invasion, extrahepatic metastasis, poor liver reserve, or multifocal disease may preclude surgery. Transplantation may be an option for select patients; however, limited resources frequently delay this intervention. Tumor ablation may be considered to bridge patients to transplantation or as definitive treatment when transplantation is contraindicated. Percutaneous thermal techniques, such as radiofrequency ablation (RFA) and microwave ablation (MWA) are widely used for HCC. These 


\section{Gastro Intestinal \\ Tumors}

\begin{tabular}{l|l}
\hline Gastrointest Tumors 2018;5:109-116 \\
\hline DOI: 10.1159/000495135 & $\begin{array}{l}\text { @ 2019 S. Karger AG, Basel } \\
\text { www.karger.com/gat }\end{array}$ \\
\hline
\end{tabular}
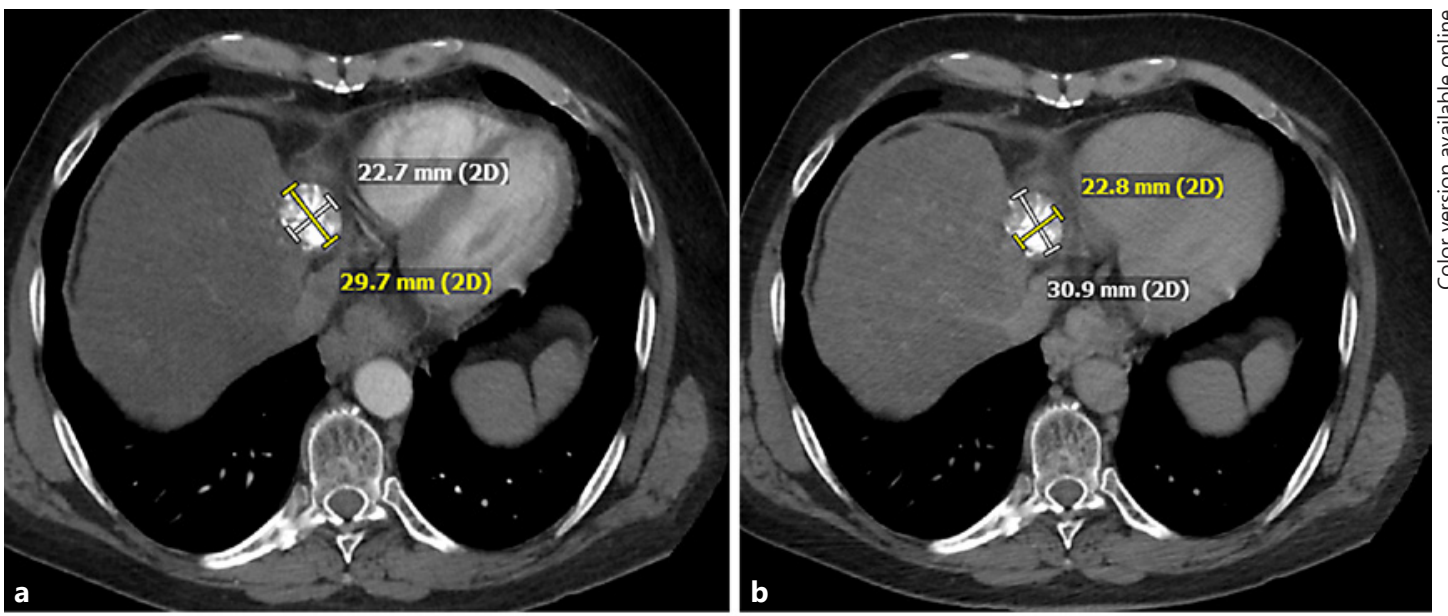

Fig. 3. One month post-procedure contrast-enhanced follow-up CT scan in arterial (a), portal venous (b), and delayed (c) phases revealing decreased tumor size $(30 \times 23 \mathrm{~mm})$, decreased arterial phase enhancement, and dense Ethiodol throughout the mass consistent with tumor ablation.
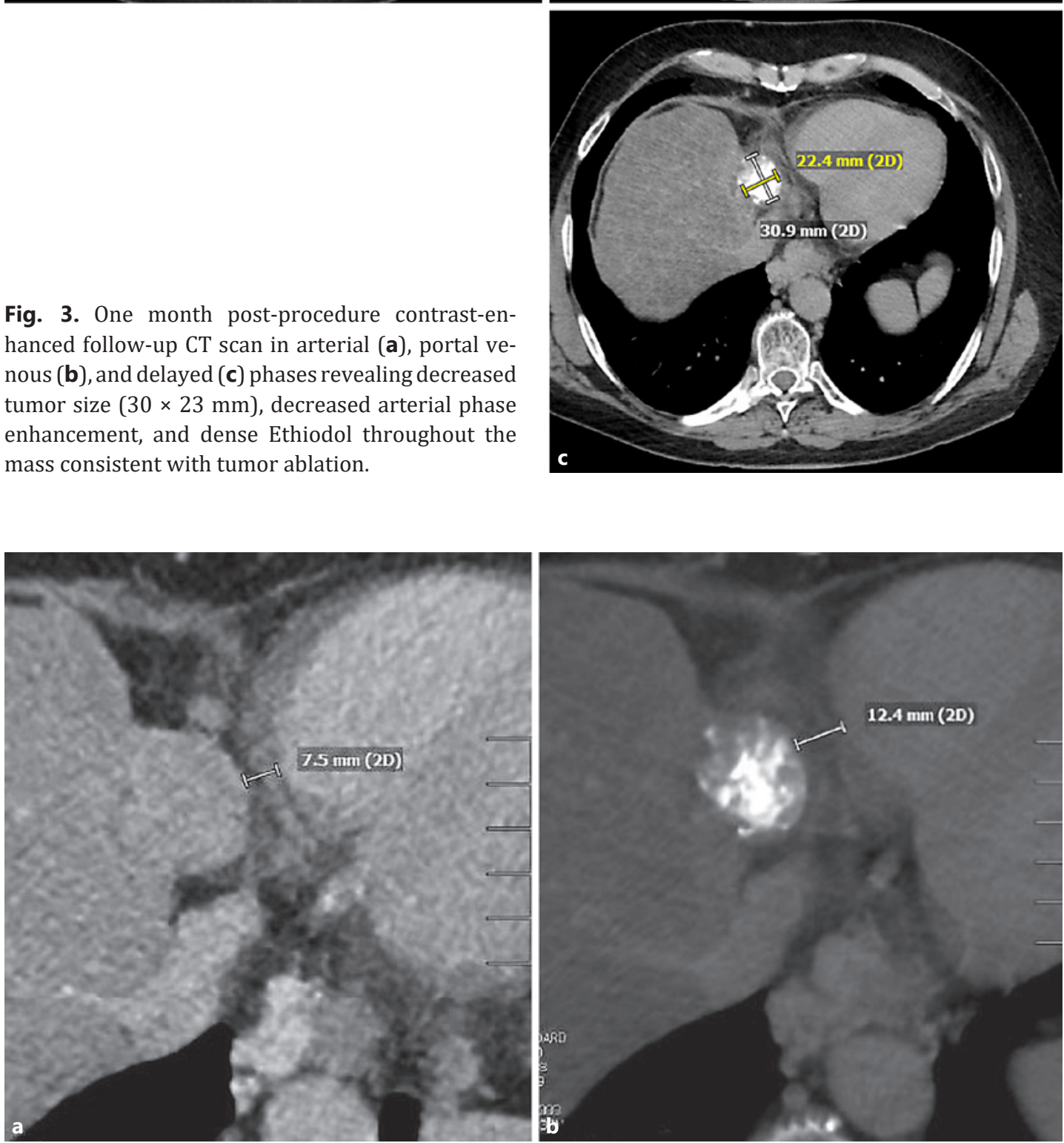

Fig. 4. a, b Comparison of pre-procedural distance $(7.5 \mathrm{~mm})$ between the tumor and the RV, and post-procedural (12.4 mm). 
techniques have been shown to provide long-term results comparable to surgical resection [11]. Percutaneous cryoablation (PC) is another technique increasingly employed for treatment of HCC; however, it may be precluded due to the vicinity of vulnerable structures as well as the "thermal sink" effect. This refers to the thermodynamic effect of flowing blood within major vessels on ice ball formation leading to incomplete ablation and subsequent local tumor recurrence [12]. This phenomenon affects thermal ablations in addition to PC, as large volumes of flowing blood will dissipate the heat generated to achieve cytotoxic temperatures at the tumor site [13]. Therefore, tumors in close proximity to high-flow vascular structures such as the heart or major vessels may be poor candidates for RFA, MWA, and CA.

Successful eradication of HCC requires that the total tumor volume and the periphery of the tumor achieve irreversible cell death to eliminate microscopic invasion and future metastasis [14]. Thus, the ablation diameter must be wider than the diameter of the lesion itself. An ablation margin of $>5 \mathrm{~mm}$ is preferred to eliminate microsatellites present at the time of treatment [15]. Indeed, when the RFA ablation margin was $>3 \mathrm{~mm}$ in 3 dimensions, local progression was $0 \%$ compared to $26.3 \%$ when this margin was not achieved. Local progression occurred at the location of the thinnest ablative margin $~ 75 \%$ of the time, and failure to achieve an adequate ablation margin was often due to "thermal sink" effect from nearby vascular structures such as hepatic arteries or portal vein [16].

Juxta-cardiac (JC) tumor ablation may be indicated for poor surgical candidates: however, there is a need to minimize collateral damage to nearby vital structures. Concerns when utilizing thermal techniques for JC lesions include injury to coronary or cardiac tissue [17], and local recurrence [18]. Additionally, multiple reports exist of pericardial tamponade complicating RFA of HCC lesions that abut or involve the pericardium [19]. Safety and efficacy of thermal ablation (RFA, MWA) for JC tumors were recently demonstrated to be comparable to non-JC tumors [20]. That study characterized lesions $<10 \mathrm{~mm}$ from the cardiac border as JC. The lesion described in this report measured $7.5 \mathrm{~mm}$ from the blood inside the RV. An ablative margin of $5 \mathrm{~mm}$ with RFA may have damaged the pericardium and the RV myocardium in this patient.

While thermal ablation has proven to be an effective form of treatment for HCC, problematic tumor location may lead clinicians to consider other techniques. Since their implementation, RFA, MWA, and PC have supplanted percutaneous ethanol injection (PEI) as the preferred modalities for percutaneous HCC ablation. PEI refers to percutaneous, intratumoral injection of highly concentrated ethanol, inducing direct damage through coagulative necrosis of tumor cells through protein denaturation, dehydration of the tumor, and microvascular ischemia. PEI has been demonstrated to be safe, effective, and inexpensive. It may be the treatment of choice for small HCC lesions particularly in resource-poor settings [21]. Potential complications of PEI include injury to the main bile duct [22], local tumor recurrence, and systemic ethanol egress resulting in cardiopulmonary collapse [23]. Multiple treatment sessions may be required, as the diffusion capacity of ethanol is limited due to concurrent inflammation, cirrhosis, and fibrotic tissue [24].

Dense cirrhotic tissue surrounding the tumor, however, may serve to limit extravasation of ethanol, containing the treatment to the desired area [25]. PEI demonstrates similar efficacy to RFA for HCC lesions $<2 \mathrm{~cm}$ and continues to be utilized for treating HCC adjacent to vulnerable structures [26, 27]. PEI is versatile: a multipronged needle with a high-dose strategy was shown to safely and effectively ablate HCC up to $5.0 \mathrm{~cm}$ in diameter. The multipronged device was implemented to avoid incomplete ablation due to intratumoral septations [28]. PEI is not affected by the "thermal sink" phenomenon, thus it can effectively treat tumors located near large vessels, such as the heart. 


\section{Conclusion}

PEI is a safe, effective, reliable and inexpensive modality to treat HCC in sensitive locations. It is not susceptible to the thermodynamic effects of rapid blood flow; therefore, it can reliably achieve adequate ablation close to large vascular structures such as the heart and great vessels. Additionally, PEI can be used to safely ablate JC tumors abutting the pericardium. It was employed in this patient to prevent incipient extrinsic cardiac compression by a rapidly expanding JC HCC.

\section{Disclosure Statement}

The authors declare no conflicts of interest.

\section{Statement of Ethics}

Informed, written consent was obtained from the patient prior to participation in this study. The case was reviewed by UF IRB-03 Chairman and it was determined that as a single case report, it does not require IRB approval.

\section{References}

1 Greten TF, Papendorf F, Bleck JS, Kirchhoff T, Wohlberedt T, Kubicka S, et al. Survival rate in patients with hepatocellular carcinoma: a retrospective analysis of 389 patients. Br J Cancer. 2005 May;92(10):1862-8.

2 A new prognostic system for hepatocellular carcinoma: a retrospective study of 435 patients: the Cancer of the Liver Italian Program (CLIP) investigators. Hepatology. 1998 Sep;28(3):751-5.

3 Kumar V, et al. Robbins and Cotran Pathologic Basis of Disease. 9th ed. Elsevier Saunders; 2015.

4 Zhu AX, Duda DG, Sahani DV, Jain RK. HCC and angiogenesis: possible targets and future directions. Nat Rev Clin Oncol. 2011 May;8(5):292-301.

5 Llovet JM, Ricci S, Mazzaferro V, Hilgard P, Gane E, Blanc JF, et al.; SHARP Investigators Study Group. Sorafenib in advanced hepatocellular carcinoma. N Engl J Med. 2008 Jul;359(4):378-90.

6 Le Grazie M, Biagini MR, Tarocchi M, Polvani S, Galli A. Chemotherapy for hepatocellular carcinoma: the present and the future. World J Hepatol. 2017 Jul;9(21):907-20.

7 Deng GL, Zeng S, Shen H. Chemotherapy and target therapy for hepatocellular carcinoma: new advances and challenges. World J Hepatol. 2015 Apr;7(5):787-98.

8 Jing Y, Han Z, Zhang S, Liu Y, Wei L. Epithelial-Mesenchymal Transition in tumor microenvironment. Cell Biosci. 2011 Aug; 1(1):29.

9 Fransvea E, Angelotti U, Antonaci S, Giannelli G. Blocking transforming growth factor-beta up-regulates E-cadherin and reduces migration and invasion of hepatocellular carcinoma cells. Hepatology. 2008 May; 47(5):1557-66.

10 Bruix J. Treatment of hepatocellular carcinoma. Hepatology. 1997 Feb;25(2):259-62.

11 Nault JC, Sutter O, Nahon P, Ganne-Carrié N, Séror O. Percutaneous treatment of hepatocellular carcinoma: state of the art and innovations. J Hepatol. 2017 Oct;S0168-8278(17)32351-6.

12 Soule E, Matteo J. Finally, a Minimally Invasive Option for Intrahepatic Inferior Vena Cava Invasion by Hepatocellular Carcinoma. Gastrointest Tumors 2018;5:54-61

13 Lau WY, Leung TW, Yu SC, Ho SK. Percutaneous local ablative therapy for hepatocellular carcinoma: a review and look into the future. Ann Surg. 2003 Feb;237(2):171-9.

14 Crocetti L, Lencioni R. Thermal ablation of hepatocellular carcinoma. Cancer Imaging. 2008 Feb;8(1):19-26.

15 Yamakado K, Takaki H, Nakatsuka A, Yamaknaka T, Fujimori M, Hasegawa T, et al. Radiofrequency ablation for hepatocellular carcinoma. Gastrointestinal Intervention. 2014;3(1):35-9.

16 Kim YS, Lee WJ, Rhim H, Lim HK, Choi D, Lee JY. The minimal ablative margin of radiofrequency ablation of hepatocellular carcinoma ( $[\{\mathrm{GT}\}] 2$ and $[\{\mathrm{LT}\}] 5 \mathrm{~cm}$ ) needed to prevent local tumor progression: 3D quantitative assessment using CT image fusion. AJR Am J Roentgenol. 2010 Sep;195(3):758-65.

17 Loh KB, Bux SI, Abdullah BJ, Raja Mokhtar RA, Mohamed R. Hemorrhagic cardiac tamponade: rare complication of radiofrequency ablation of hepatocellular carcinoma. Korean J Radiol. 2012 Sep-Oct;13(5):643-7.

18 Yang W, Yan K, Wu GX, Wu W, Fu Y, Lee JC, et al. Radiofrequency ablation of hepatocellular carcinoma in difficult locations: strategies and long-term outcomes. World J Gastroenterol. 2015 Feb;21(5):1554-66. 
19 Chun JY, Ho CS. Management of Pericardial Effusion following Cardiac Perforation during Radiofrequency Ablation of Hepatocellular Carcinoma. Semin Intervent Radiol. 2014 Mar;31(1):101-3.

20 Kwan J, Appuhamy C, Lim GH, Huang IK, Quek L, Pua U. Safety and Efficacy of Percutaneous Thermal Ablation of Juxta-Cardiac Hepatic Tumours. Cardiovasc Intervent Radiol. 2018 Jun;41(6):920-7.

21 Omata M, Cheng AL, Kokudo N, Kudo M, Lee JM, Jia J, et al. Asia-Pacific clinical practice guidelines on the management of hepatocellular carcinoma: a 2017 update. Hepatol Int. 2017 Jul;11(4):317-70.

22 Cha DI, Lee MW, Rhim H, Choi D, Kim YS, Lim HK. Therapeutic efficacy and safety of percutaneous ethanol injection with or without combined radiofrequency ablation for hepatocellular carcinomas in high risk locations. Korean J Radiol. 2013 Mar-Apr;14(2):240-7.

23 Naik B, Matsumoto AH. Acute cor pulmonale and right heat failure complicating ethanol ablative therapy: anesthetic and radiologic considerations and management. Cardiovasc Intervent Radiol. 2013 Oct;36(5): 1213-20.

24 Ansari D, Andersson R. Radiofrequency ablation or percutaneous ethanol injection for the treatment of liver tumors. World J Gastroenterol. 2012 Mar;18(10):1003-8.

25 Livraghi T, Benedini V, Lazzaroni S, Meloni F, Torzilli G, Vettori C. Long term results of single session percutaneous ethanol injection in patients with large hepatocellular carcinoma. Cancer. 1998 Jul;83(1):48-57.

26 Lin SM, Lin CJ, Lin CC, Hsu CW, Chen YC. Radiofrequency ablation improves prognosis compared with ethanol injection for hepatocellular carcinoma $[\{\mathrm{LT}\}]$ or $=4 \mathrm{~cm}$. Gastroenterology. 2004 Dec;127(6):1714-23.

27 McWilliams JP, Yamamoto S, Raman SS, Loh CT, Lee EW, Liu DM, et al. Percutaneous ablation of hepatocellular carcinoma: current status. J Vasc Interv Radiol. 2010 Aug;21(8 Suppl):S204-13.

28 Kuang M, Lu MD, Xie XY, Xu HX, Xu ZF, Liu GJ, et al. Ethanol ablation of hepatocellular carcinoma Up to $5.0 \mathrm{~cm}$ by using a multipronged injection needle with high-dose strategy. Radiology. 2009 Nov;253(2):552-61. 